\title{
Necesidades específicas de apoyo educativo en el alumnado con capacidad intelectual límite: aspectos para su intervención psi- copedagógica
}

\section{Specific needs in educational support for students with borderline intellec- tual capacity: factors for psychoeducational intervention}

\author{
Diego Jesús Luque-Parra* \\ Eduardo Elósegui-Bandera \\ Dolores Casquero-Arjona \\ Universidad de Málaga, España \\ (Rec.: diciembre de 2014 - Acept.: abril de 2016)
}

\begin{abstract}
Resumen
Los niños y niñas con capacidad intelectual límite presentan unas necesidades específicas de apoyo educativo. Estas están centradas en el desarrollo de la memoria de trabajo con sus asociaciones a lo perceptivo-atencional y al lenguaje, así como en la mejora de los aprendizajes lectoescritor y de razonamiento matemático, debiendo ser reforzado su pensamiento estratégico. En este artículo, se hace un primer acercamiento conceptual y definitorio de la capacidad intelectual límite, situándolo en un ámbito psicopedagógico, estableciendo de forma general sus posibles dificultades de aprendizaje. En una segunda parte, se determinan las necesidades específicas que este alumnado plantea, haciéndose algunas consideraciones sobre la evaluación psicopedagógica las cuales se encuentran fundamentadas en el conocimiento del caso (alumno y contextos), mediante una propuesta de adecuación a ese alumno y con objetivos de compensación y favorecimiento del desarrollo de sus capacidades. Por último, de acuerdo al análisis de la revisión teórica, se concluye que la intervención psicopedagógica en el alumnado con capacidad intelectual límite debe realizarse desde un análisis profundo y riguroso de sus características personales y de contexto. De este modo, los resultados de la evaluación psicopedagógica se convierten en necesidades específicas de apoyo educativo. Todo ello en un ámbito de inclusión social y educativa y desde criterios de normalización.
\end{abstract}

Palabras clave: capacidad intelectual límite, intervención, necesidades específicas de apoyo educativo, respuesta educativa, programas de refuerzo educativo.

\begin{abstract}
Children with borderline intellectual functioning present specific needs of educational support. These are focused on the development of work memory associated with perception, attention and language, as well as the improvement of reading and writing skills and mathematic reasoning, enhancing strategic thinking. This paper attempts to provide a general conceptual framework about borderline intellectual functioning, from an educational psychology approach, establishing thus possible learning difficulties from a general perspective. Moreover, the specific needs experienced by these students are determined, taking into account some considerations about psychoeducational assessment that are based on case study (student and contexts), through an educational proposal for this student, which includes compensation objectives and strengthening of their capacities. Finally, according to the literature review, it is concluded that psychoeducational intervention on students with borderline intellectual functioning, must be carried out from a thorough analysis of their personal and contextual characteristics. In this way, the results of psychoeducational assessment become specific needs of educational support, in a framework of socio-educational inclusion and standardization criteria.
\end{abstract}

Keywords: borderline intellectual functioning, educational intervention, specific needs of educational support, educational response, programs of educational reinforcement..

\footnotetext{
* Correspondencia a: Diego Luque Parra. Departamento de Psicología Evolutiva y de la educación. Dirección postal: Universidad de Málaga. Campus de Teatinos (29071), Málaga. Email: dluque@uma.es.
} 


\section{Introducción}

Cuando se cita o habla del término capacidad intelectual límite (en adelante CIL), suele referirse a un concepto o categoría diagnóstica de características parecidas a la discapacidad intelectual, pero de menor gravedad; concepto con el que se asume una entidad explicativa de posibles limitaciones personales, sociales, laborales y, en el ámbito educativo, de dificultades de aprendizaje (Elósegui, Luque \& Casquero, 2012). Ciertamente, como han expresado ya Artigas-Pallarés, Rigau-Natera y García-Nonell (2007), Luque, Elósegui y Casquero (2014), entre otros, se trata de un diagnóstico impreciso e inestable que posee una menor consistencia en sus factores de conjunto y es definido sobre el intervalo de inteligencia o $\mathrm{Cl}$ entre 70 y 85 . En todo caso, parece estar claro que el concepto de CIL apuntaría a la mayor probabilidad o predisposición a tener dificultades de aprendizaje, adaptación o interacción social sobre la base de una capacidad intelectual levemente inferior a la media poblacional.

En consonancia con esa probabilidad de limitaciones, los alumnos y alumnas con capacidad intelectual límite tienen necesidades específicas de apoyo educativo (en adelante, NEAE), al presentar dificultades de aprendizaje o alguna limitación en su desarrollo personal y social. De este modo, se pone en evidencia la necesidad de una atención no distintiva, sino en justa distribución a sus características personales, a través de una respuesta individualizada y dentro de un ámbito de normalización y de inclusión. Esto es así porque con el término de neaes no se alude a un conjunto o grupo homogéneo por su adjetivación de específico, sino que como concepto engloba a situaciones e historias personales y sociales diferentes y únicas. En este sentido, al igual que el resto del alumnado, tienen las mismas necesidades educativas y estas se relacionan básicamente con: a) las características de la persona a las que se le añade su limitación o dificultad; b) la adecuación en sus procesos de enseñanza-aprendizaje, y c) los elementos de aceptación, comprensión y apoyo de la comunidad (Luque \& Rodríguez, 2006; Luque \& Luque-Rojas, 2015).

Desde este núcleo de relaciones, adquiere sentido una educación para la diversidad que contemple necesariamente la multiplicidad de personas y sus circunstancias personales y sociales, sus capacidades, intereses, motivaciones y aprendizaje. Así pues, debe considerarse una respuesta educativa y oferta pedagógica en adecuación y ajuste.

En este artículo, se trata de analizar las necesidades específicas de apoyo educativo que los alumnos y alumnas con CIL presentan. Ello a partir de las dificultades que pueden tener en sus procesos de enseñanza-aprendizaje, con carácter temporal o duradero, y precisando de recursos educativos específicos. Des- de esta concepción, aceptamos la CIL como entidad diagnóstica, pero tratando de evitar el efecto de un etiquetaje (que suele disminuir, cuando no anular, las posibilidades de enseñanza - aprendizaje) y convirtiendo la evaluación y diagnóstico en un punto de partida de la intervención psicopedagógica (no de llegada). Del mismo modo, perseguimos objetivos de desarrollo curricular y académico, pero con fines de desarrollo personal y social.

El trabajo se estructura en tres partes. En la primera se hace un acercamiento definitorio a la capacidad intelectual límite, en su asociación a las dificultades de aprendizaje para, en la segunda, continuar con un análisis de las necesidades específicas que estos alumnos y alumnas pueden presentar. En un tercer apartado, se discute finalmente sobre algunas reflexiones acerca de la respuesta educativa a esas necesidades. En suma, se pretende hacer una revisión de la intervención psicopedagógica con este alumnado, tratando de contribuir a su mejor conocimiento y situación diagnóstica, así como de favorecer la conveniente reflexión-acción en el profesorado en sus casos.

\section{Alumnado con capacidad intelectual límite}

\section{Aspectos conceptuales y Definitorios}

En general, las teorías psicológicas que han fundamentado el desarrollo educativo, particularmente en la Educación Especial, han conceptualizado la mente como una capacidad de dominio general. Así, podemos entender por inteligencia una capacidad mental general necesaria para el pensamiento, la actuación con intencionalidad, el aprendizaje, la comprensión y la adaptación al entorno. Con esta definición se aúnan puntos de vista teóricos en una integración de inteligencia general con factores o habilidades, de acuerdo con el modelo triárquico de Sternberg (1988) y el de Greenspan y cols. (Greenspan \& Love, 1997; Greenspan, Switzky \& Granfield, 1996). Esto es, una integración de inteligencia conceptual, inteligencia práctica e inteligencia social. Consecuentemente, cualquier limitación o disfunción de esa capacidad intelectual general, resultará en un trastorno intelectual del desarrollo.

Siendo obvia la dificultad y la importancia de su medición, aceptamos que la evaluación de la inteligencia por pruebas de $\mathrm{Cl}$ sigue siendo la mejor forma de valorar el funcionamiento intelectual; ello siendo conscientes de que en ocasiones las pruebas de $\mathrm{Cl}$ se aplican de forma masiva, descontextualizada o con abundancia de academicismos en contenidos y objetivos. De todas formas, aceptamos que el $\mathrm{Cl}$ es una estimación fiable de la inteligencia (como capacidad general) y, en consecuencia, un buen criterio para el diagnóstico de una discapacidad o de un menor funcionamiento intelectual, al cual se le añade el manejo y desenvolvimiento que tiene la persona en su relación con el contexto 
próximo y las habilidades que desarrolla.

La capacidad intelectual límite (CIL) es la manifestación de un estado particular de funcionamiento cognitivo en el extremo menor de la curva normal de la inteligencia ( $\mathrm{Cl}$ de entre 70 y 85), con alguna limitación o disfunción en su capacidad de adaptación, lo que no impide un aceptable grado de autonomía en las actividades de la vida diaria. Este estado puede explicarse desde alguna alteración o disfunción neurológica que provoca retrasos y alteraciones en el desarrollo de funciones psicológicas directamente implicadas en el aprendizaje y en la adaptación a los contextos.

Aunque la CIL no es considerada como un criterio diagnóstico, es mantenida sin embargo, como categoría diagnóstica en la que se engloban individualidades con notables diferencias psicológicas entre ellas. En este sentido, en el Manual Diagnóstico y Estadístico de los Trastornos Mentales (DSM-IV-TR; American Psychological Association [APA], 2002; DSM-5; American Psychological Association [APA], 2014), la CIL es clasificada en la sección de Otros problemas que pueden ser objeto de atención clínica, dentro de la que se establece como problema adicional, cuidando el diagnóstico diferencial con la discapacidad intelectual y su particular coexistencia con otros trastornos mentales que pudieran darse. Así, la CIL ha sido estudiada a partir de sus características definitorias (Bonnifaci \& Snowling, 2008; Shaw, 2008; Singh, 2004; Verguts \& De Boeek, 2001; Vuijk, Hartman, Scherder \& Visscher, 2010); en la búsqueda de sus bases explicativas (Haier, White \& Alkire, 2003; Morton, 2004) y también, desde su funcionamiento cognitivo a través de la función ejecutiva (Alloway, 2010) o la memoria de trabajo (Hartman, Houwen, Scherder \& Visscher, 2010; Schuchardt, Gebhardt \& Mäehler, 2010; entre otros). Ofrece igualmente interés una aproximación psiconeurológica (Artigas-Pallarés, 2003; Artigas-Pallarés et al., 2007; Narbona \& Crespo-Eguílaz, 2005; Tirapu-Ustarroz \& Muñoz-Céspedes, 2005), así como la evaluación e intervención psicoeducativa en las dificultades de aprendizaje (Elósegui et al., 2012; Luque \& Rodríguez, 2006; Luque et al., 2014; Romero \& Lavigne, 2005).

En todos estos estudios recientes, revisados con criterios de diagnóstico e intervención psicoeducativa y pedagógica, podrían establecerse varios elementos comunes de acuerdo con el acercamiento de los autores a la capacidad intelectual límite. En primer lugar, estos estudios participan de la concepción de la CIL como una categoría diagnóstica que situándose a una distancia de la discapacidad intelectual, explica las posibles limitaciones cognitivas, escolares, sociales o laborales, con algunas similitudes a las de aquella, pero con un aceptable grado de autonomía en las actividades de la vida diaria. En segundo lugar, tanto el funcionamiento cognitivo en el extremo menor de la curva normal ( $\mathrm{Cl}$ de entre 70 y 85 ), como las dificultades que puedan darse, son explicados desde alguna alteración o disfunción neurológica que provoca retrasos y alteraciones en el desarrollo de funciones psicológicas directamente implicadas en el aprendizaje y la adaptación al medio. En tercer lugar, estos estudios también se caracterizan por su prudencia en la evaluación, requiriéndose una cuidada elaboración de la información disponible, para poder diferenciar la discapacidad intelectual leve, de la capacidad intelectual límite.

Finalmente, también existiría acuerdo entre algunos autores (Artigas-Pallarés et al., 2007; Schuchardt et al., 2010; Vuijk et al., 2010) en que, al igual que en la discapacidad intelectual, no existen características del comportamiento o de personalidad que sean típicas o asociadas unívocamente a las personas con CIL. Es por ello que pueden encontrarse personas pasivas o dependientes, así como otras más activas o independientes e incluso impulsivas. En suma, aquello que precisamente determina su carácter de límite es lo que le confiere un diagnóstico impreciso o inestable, al no asociársele otros aspectos o condiciones adicionales; de ahí que sea necesario acudir a factores individuales, sociales y de historia familiar (conformados en una integración conceptual) para explicar determinadas conductas y desarrollos individuales, y predecir o prevenir dificultades o limitaciones.

En suma, el valor conceptual de la CIL estaría situado en un núcleo de predisposición a tener dificultades en los aprendizajes e interacción social, aceptándose como una causa subyacente, la capacidad de inteligencia en el límite inferior a la media poblacional (Artigas-Pallarés et al., 2007; Luque et al., 2014).

\section{Dificultades de aprendizaje y capacidad intelectual límite}

Como se ha expresado (Luque \& Rodríguez, 2006; Romero \& Lavigne, 2005), los niños y niñas con CIL ofrecen un análisis de mayor complejidad tanto por la variedad de sus funcionamientos cognitivos (posibles limitaciones) como por las dificultades o falta de estrategias y habilidades a las que se unen la historia familiar, factores sociales y de contexto. En cualquier caso, tienen una elevada probabilidad de dificultades de aprendizaje (Cuadro 1), por lo que se hace obvio expresar que la detección e intervención tempranas puede proporcionar una adaptación y desarrollo acordes con sus características o limitaciones, para un futuro personal, social y laboral digno y productivo en la sociedad.

A este respecto, nos referimos con el término Dificultades de Aprendizaje a su conceptuación como trastornos intrínsecos al alumno, debidos presumiblemente a una alteración o disfunción psiconeurológica que provoca retrasos en el desarrollo de funciones psicológicas (como por ejemplo el lenguaje, la atención, la memoria de trabajo o procedimientos de regulación del 
pensamiento) directamente implicados en el aprendizaje (Romero \& Lavigne, 2005). Desde este punto de vista, y desde un marco de intervención psicoeducativa, los niños y niñas con CIL se situarían en el extremo de un continuo diagnóstico en el que la gravedad, cronicidad y dificultad del aprendizaje serían de máxima entidad, sirviendo de antesala a la discapacidad intelectual (Luque \& Rodríguez, 2006).

Cuadro 1. Dificultades de aprendizaje en el alumnado con Capacidad Intelectual Límite

- Dificultades en psicomotricidad fina.
- Dificultades de tipo lingüístico (Propensión a di-
ficultades articulatorias; uso de estructuras sen-
cillas a nivel morfosintáctico; menor desarrollo
semántico; uso del lenguaje más funcional y
usual, con menor grado de abstracción).

- Distracción y menor capacidad de atención (selectiva, sostenida y dividida).

- Menor capacidad creativa y capacidad de adaptación a situaciones nuevas.

- Niveles de aprendizaje lentos, con necesidad de apoyos.

- Dificultades de aprendizaje de lectura (calidad y comprensión lectoras).

- Dificultades de la expresión escrita (ortografía y composición).

- Dificultades de aprendizaje matemático (razonamiento y resolución de problemas)...

- Necesidad de estrategias para la organización, planificación y desarrollo de tareas.

- Dificultades en la planificación y organización de espacio y tiempo.

- Baja autoestima e inseguridad.

- Baja tolerancia al fracaso y la frustración.

- Algunas dificultades en decisiones y resolución de conflictos.

Nota: Adaptado de Romero y Lavigne (2005) y Luque y Rodríguez (2006).

Quedando esta posición clara, no lo es tanto en sus intervenciones y respuesta educativa, ya que la posición dentro de un intervalo de $\mathrm{Cl}$, como criterio psicométrico, no es explicativa de las realidades individuales y de sus relaciones contextuales. En este sentido, tanto Pallisera (2003) como el Gobierno de Aragón (2008) expresan que los niños y niñas con CIL son los desubicados de la escuela y de las propuestas del sistema educativo, por cuanto no perteneciendo estrictamente al ámbito de acción de la educación especial, sus pro- pias dificultades de aprendizaje pueden ser de mayor gravedad y cronicidad que las de otros alumnos con dificultades específicas de aprendizaje, trastornos de déficit atencional con hiperactividad (TDAH), dificultades específicas de lectura y escritura o dislexia.

Así, el alumnado con $\mathrm{CIL}$, aunque pueda coincidir con otras dificultades específicas de aprendizaje en el desarrollo fonológico o en determinados aspectos cognitivos, será su $\mathrm{Cl}$ más bajo que la media el elemento diferenciador y definitorio. De esta forma sus dificultades de aprendizaje podrían explicarse desde un funcionamiento cognitivo con déficits en las funciones perceptivas, atencionales, de memoria y razonamiento, así como en sus relaciones y procesos y en las estrategias de afrontamiento, canalización y elaboración de la información. Este déficit que no solo se sitúa en una estructura, sino en sus parámetros de velocidad y eficacia, valorándose limitaciones o estrategias a aplicar menos adecuadas. Dado que los niños y niñas con CIL muestran una gran variabilidad en su ejecución de tareas, puede decirse que se diferenciarían de los niños y niñas con desarrollo normal, no tanto cuando llevan a cabo su mejor nivel, sino en su peor ejecución (Alloway, 2010).

De forma operativa, en el estudio del aprendizaje lectoescritor podríamos establecer que los niños y niñas con CIL responden más lentamente o con menos precisión, a tareas que implican tiempos de reacción, que los niños con cociente intelectual dentro del rango normal, independientemente de si son o no buenos lectores (Bonifacci \& Snowling, 2008). Cuando se comparan alumnos con dificultades específicas de lectura o dislexia con niños con CIL, estos tienen una velocidad de procesamiento menor a aquellos, proponiéndose esta como causa en la dificultad de la lectura (Catts, Gillispie, Leonard, Kail \& Miller, 2002) y en consonancia, con la hipótesis de una asociación entre las limitaciones de procesamiento y de eficiencia con un bajo $\mathrm{Cl}$ (Anderson, 1992; Jensen, 1998; Alloway, 2010).

Aunque un $\mathrm{Cl}$ bajo o límite pueda considerarse la base explicativa de un rendimiento bajo o lento en el aprendizaje en general y en el de la lectoescritura en particular, es importante señalar el peso que tienen los aspectos psicolingüísticos (percepción y comprensión auditiva, memoria, razonamiento y expresión verbales como conocimiento de palabras y formación de conceptos). Estos aspectos pueden valorarse en los índices de la Escala WISC-IV. En efecto, los alumnos con CIL, al igual que otros con DEAs, no se caracterizan tanto por una menor memoria de trabajo (MT) y velocidad de procesamiento (VP) de la Escala (capacidades de memoria, de atención y agilidad de procesamiento) como por una menor habilidad en el mantenimiento de estímulos (grafemas y fonemas en el aprendizaje lectoescritor), así como en una baja eficacia de tareas ejecutivas en el procesamiento de la información. 
Esta menor habilidad implica una no automatización de los procesos de identificación y reconocimiento de estímulos, lo que explicaría que el alumno se vea obligado a invertir más tiempo (prestar mayor atención) del necesario en identificar y reconocer; pudiendo simultáneamente olvidar algunos de los estímulos que ya había reconocido antes. De esta manera, la automatización de los procesos básicos (reconocimiento de letras, sílabas, palabras, números; hacer la conversión grafema-fonema o fonema-grafema) estaría en la base explicativa de una práctica lectora más lenta y menos precisa sobre unos aspectos de desarrollo fonológico ya adquiridos y conformados (Elósegui et al., 2012).

Como se ha expresado en un estudio (Elósegui, Luque \& Casquero, 2011), en los niños y niñas con CIL, se pondría de manifiesto que la MT es, más que un sistema de memoria, un sistema atencional operativo que trabaja con contenidos de la memoria, quedando en un sistema central de control cognitivo y de procesamiento ejecutivo en sus interacciones entre las diversas funciones y procesos mentales (atención, percepción, motivación, emoción y memoria). De ahí que pueda observarse que los alumnos con CIL presentarían más dificultades en el procesamiento visoperceptivo y el control de la información (puntuaciones de MT y VP en la Escala WISC-IV) en su relación con el aprendizaje lectoescritor, que en aspectos de desarrollo lingüístico; toda vez que el nivel ejecutivo guardaría relación con la automatización de los procesos visoperceptivos (un control ejecutivo con menor capacidad de inhibición de la información irrelevante y lentitud en procesos mentales de razonamiento). De esta manera, podría expresarse que los niños con CIL serían más inatentos por su dificultad en seguir aprendizajes cuya comprensión, procesamiento y ejecución, no resultan eficaces, debido no tanto a una valoración genérica de su menor inteligencia, sino al funcionamiento menos estratégico que aquella implica.

Para algunos autores (Morgado, 2005; Schuchardt et al., 2010) existiría una interrelación dinámica entre la memoria de trabajo, la velocidad de procesamiento y el razonamiento, quedando la velocidad de procesamiento de los niños con CIL como una compensación ejecutiva, frente a una reducción en el uso de la memoria de trabajo. De acuerdo con ello, en una evaluación de la inteligencia con la WISC-IV, tanto los aspectos de $\mathrm{Cl}$ Total como los de VP y de MT, aportarían una base explicativa en la transformación y manejo mental de la información, el razonamiento fluido o las habilidades y estrategias para los aprendizajes, pero también, que estas capacidades y sus indicadores no son potentes predictores en el éxito del aprendizaje lectoescritor. De esta manera sería conveniente la introducción de medidas directas de la capacidad de aprendizaje del alumno en las que podría considerarse los aspectos de calidad, velocidad y comprensión lectoras como ele- mentos de logro de objetivos en ese aprendizaje.

Aunque en la evaluación psicológica y pedagógica de las dificultades de aprendizaje se tienen criterios definitorios y procedimientos varios, parece existir acuerdo en que la respuesta o actuaciones en los procesos de enseñanza-aprendizaje pueden tenerse dos marcos de intervención. Uno centrado en actuaciones sobre procesos básicos que, una vez obtenidos resultados en el aprendiz, pueden transferirse al proceso de enseñanza-aprendizaje en habilidades académicas. $Y$ un segundo marco de intervención en el que se obtiene un desarrollo de enseñanza-aprendizaje de las habilidades académicas partiendo de un conjunto integrado desde el que se corrigen y entrenan habilidades cognitivas e instrumentales. En cuanto a resultados o productos de rendimiento de aprendizaje, los programas de intervención en procesos básicos, han tenido resultados poco eficaces, al no conseguir en el alumnado con Dificultades de Aprendizaje, una mejora de su nivel. Esto debido, básicamente, a que no se producen los efectos de transferencia y generalización supuestos o esperados. Por el contrario, podría confirmarse que los alumnos con Dificultades de Aprendizaje mejoran tras una intervención específica en estrategias de aprendizaje o de pensamiento, configurándose así una respuesta educativa a la realidad concreta del alumnado (Luque, 2006). Esta respuesta pues, nos induce a reflexionar no tanto sobre las dificultades del individuo como en las necesidades que plantean, además del grado de especificidad asociado.

\section{Necesidades Específicas de Apoyo Educativo del Alumnado con Capacidad Intelectual Límite}

Comenzaremos precisando que el término NEE es un constructo que abarca más que su simple conceptuación operativa de cara al alumnado con determinados apoyos y atenciones educativas específicas (derivadas de discapacidad o trastornos graves de conducta) y recoge toda una filosofía educativa de personalización, normalización e inclusión. A su vez, constituye una concepción aplicada de respuestas para satisfacer necesidades. Se entiende que las nees no poseen una entidad diagnóstica, pero sí de valoración.; no constituyen un concepto distintivo o negativo, sino de especificidad para su tratamiento, $y$, finalmente, no es un adjetivo sustantivado, sino un término expresivo a una respuesta de calidad y con equidad al alumnado que las precisa (cuadro 2).

Este concepto de necesidades educativas especiales, desde mediados de la década de 2000 , pasa a convertirse en una entidad más dentro de las categorías de la atención educativa al alumnado, diferente a la ordinaria. Así, se establece una estructura de valoración educativa en la que el término nees, se circunscribe al alumnado con determinados apoyos y atenciones educativas específicas derivadas de discapacidad o tras- 
tornos graves de conducta o, lo que es lo mismo, aquel que requiera, por un periodo de su escolarización o a lo largo de toda ella, determinados apoyos y atenciones educativas específicas derivadas de esas circunstancias. Ciertamente es una concepción restringida, con riesgo de convertir la discapacidad ( $\mathrm{y}$ al mismo concepto de nees) en entidad diagnóstica, repitiendo patrones de deficiencia o limitación (Luque, 2009). A pesar de esa categorización, pensamos que, desde una perspectiva pedagógica, el término de necesidades educativas especiales tiene una amplia aplicación, ya que encierra toda una filosofía de valoración, ni distintiva ni negativa, sino de especificidad para una respuesta de calidad y con equidad al alumnado que las presente (visión operativa de actuación y recursos en un ámbito escolar).

Cuadro 2. Algunas características de las necesidades educativas especiales

- El adjetivo especial se da por razones de énfasis en la atención sobre las necesidades educativas que comparten con el resto del alumnado. No son especiales por trastorno o distintividad, sino por una mejor y específica respuesta educativa al alumno en cuestión.

- Hacen alusión a salvar obstáculos o vencer limitaciones tanto personales como contextuales, de forma que las necesidades educativas que el alumno tiene sean satisfechas con una respuesta adecuada.

- Persiguen una respuesta de carácter reforzador o compensador que equilibre la atención educativa que comparten con el resto de alumnos.

- No son una entidad diagnóstica, sino de valoración psicopedagógica hacia una adopción de medidas adecuadas a la persona y situación.

- Hacen referencia a cualquier necesidad individual, de contexto y circunstancias, en la que se precise individualización o respuesta oportuna. No tiene adjetivos añadidos en función de un diagnóstico, aunque se asocie a este por su afinidad o tipos de intervención.

Nota: Adaptado de Luque (2009).

En términos generales, la adecuación de la respuesta educativa a las necesidades del alumnado puede hacerse desde dos grandes entidades; de un lado, aqueIla en la que el desarrollo ordinario del currículum se ajusta a las necesidades que se presentan, y por otro, aquella en la que se destacan las necesidades específicas de apoyo educativo, haciendo referencia a los alumnos y alumnas que requieran una atención educativa diferente a la ordinaria por presentar necesidades educativas especiales, por dificultades específicas de aprendizaje, por altas capacidades intelectuales, por circunstancias socioculturales o de desventaja, o bien por condiciones personales o de historia escolar. El objetivo es que todos los alumnos puedan alcanzar el máximo desarrollo posible de sus capacidades personales, intelectuales, sociales y emocionales. Esta atención debe tener un carácter integral, iniciándose desde el mismo momento en que dicha necesidad sea identificada y se regirá por los principios de normalización e inclusión.

De acuerdo a esta estructura de respuesta educativa, la atención al alumnado con CIL se situaría entre las dificultades específicas de lectura y escritura o TDAH y aquellas que presentan los niños y niñas con discapacidad intelectual, resultando en un análisis y planificación con cierto nivel de complejidad. Se hace necesaria, pues, una valoración psicológica y pedagógica que aporte el mejor conocimiento de la persona y fundamente cualquier intervención educativa (cuadro 3).

Cuadro 3. Aspectos a considerar en una valoración psicopedagógica del alumnado con necesidades específicas de apoyo educativo

1. ASPECTOS PREVIOS

- Conocimiento del caso y fuentes de información. Alumnado y contextos.

- Planificación y estructura de la evaluación. Análisis y puesta a punto de los elementos de evaluación, persiguiendo la adaptación de las técnicas y procedimientos a la persona y a la situación.

2. EVALUACIÓN PSICOLÓGICA Y PEDAGÓGICA

2.1. Planificación de aspectos a evaluar:

- Desarrollo psicomotor.

- Comunicación y lenguaje.

- Capacidad intelectual y aptitudes.

- Autonomía personal y social.

- Competencia curricular.

2.2. Elementos contextuales:

- Recursos materiales y humanos.

- Accesibilidad (en general).

- Análisis y respuesta del Proyecto Educativo del Centro.

2.3. Integración de información. Propuestas de intervención.

- Valoración y diagnóstico que procedan.

- Informe o dictamen de escolarización.

- Elementos de reflexión sobre la adaptación curricular.

3. ASPECTOS DE INTERVENCIÓN

- Adaptación curricular (Grado de significatividad).

- Programas de refuerzo.

- Asesoramiento al profesorado.

- Evaluación y seguimiento.

Nota: Adaptado de Trianes y Luque (2013).

Estas consideraciones son necesarias en cualquier 
evaluación, pero lo son más aún para las personas con limitaciones, ya que un efecto secundario de las evaluaciones psicológicas y pedagógicas es la posibilidad de su conversión en un mero análisis de deficiencias o limitaciones (como consecuencia de un trastorno o alteración) a las que aplicar el tratamiento oportuno. Antes bien, el propósito de cualquier valoración debe de ser el de determinar el estado o situación del alumnado, y dadas unas determinadas dificultades, apreciarlas no tanto como problema, sino como objeto de soluciones y más aún de satisfacción de necesidades (sin basarse en la deficiencia, ni acentuar las limitaciones). Por lo tanto, la organización de la atención educativa al alumnado con CIL ha de ser fundamentada en el conocimiento del caso (alumno y contextos), a lo que ha de seguir una estructura y planificación de la evaluación, terminando en una propuesta de adecuación a ese alumno y a su realidad personal y social.

El abordaje de la respuesta educativa se llevará a cabo desde la adecuación a las necesidades y características de la persona, compensando y favoreciendo el desarrollo de sus capacidades con los recursos o medidas oportunos. En el caso del alumnado con CIL, esta adecuación tanto en la intervención en sus dificultades como en su prevención y desarrollo evolutivo debería dar respuesta, de manera general, a las necesidades de:

- Estimulación y desarrollo psicomotor tanto en la motricidad gruesa como en la motricidad fina, sobre todo.

- Desarrollo sensorial de la percepción visual, coordinación visomotora, percepción auditiva y percepción corporal.

- Desarrollo cognitivo a través de técnicas y estrategias de trabajo intelectual, potenciando las funciones de atención, memoria y razonamiento.

- Comunicación y lenguaje, mejorando la función pragmática en general, la comprensión y expresión del lenguaje o la intervención concreta en los aspectos sintácticos y semánticos.

- Desarrollo socioafectivo, interviniendo en las relaciones con los iguales, así como en la identificación y expresión de emociones.

- Refuerzo del aprendizaje lectoescritor, reforzando procesos cognitivo-perceptivos (auditivos y visuales) y estrategias de aprendizaje.

- Refuerzo de áreas académicas.

- Adecuación social y autonomía personal.

A riesgo de ser genéricos y considerando que cualquier atención al alumnado con nees es individualizada, se puede establecer un conjunto integrado de posibles necesidades educativas para personas con limitaciones o discapacidad de cualquier tipo, de forma que posteriormente, en un nivel operativo, pudiera servir de referencia para casos particulares (véase anexo: Necesidades Educativas Especiales: Desarrollo de capacidades y competencias básicas.).

\section{Respuesta educativa a las necesidades específicas de apoyo del alumnado con capacidad intelectual límite}

El principio básico de la intervención psicopedagógica es la atención educativa centrada en el reconocimiento de la diversidad del alumnado y en el favorecimiento de un entorno educativo capaz de generar esa atención. Ello considerando al alumno desde la perspectiva de sus necesidades educativas, fijando una atención en su individualidad, pero cargando sobre todo en los niveles de respuesta educativa. Desde esa atención (intervención adecuada a necesidades y contextos) debe aceptarse que la intervención psicopedagógica es un instrumento y vía de desarrollo práctico, con resultados en el proceso de enseñanza-aprendizaje del alumnado y que discurre a través de la integración con soluciones de normalización.

De forma operativa, el profesor y el equipo educativo al hacer intervenciones con alumnos con CIL para dar respuesta a sus necesidades deben adoptar una perspectiva de acción tutorial, esto es, una visión educadora y no meramente instructora, que no solo se ocupe de los programas de trabajo académico, sino que se preocupe de los procesos formativos. Se trata de favorecer un desarrollo educativo que contemple las características individuales y acepte las distintas características personales del alumnado y sus circunstancias, aportando la respuesta apropiada a su singularidad. Esta adecuación, que sin duda es de aplicación general a todo el alumnado, se hace particularmente ajustada a los que presentan CIL. Como se ha comentado para los alumnos con discapacidad intelectual (Luque \& Luque-Rojas, 2012), la personalización es lo que da sentido y significación a cualquier valoración, ya que esta solo adquirirá entidad para el conocimiento y la intervención cuando el término (adjetivo) CIL se traslade al término (sustantivo) de persona. Cuestión sobre el diagnóstico que no es baladí para la reflexión y actuación docentes puesto que en ella se encierran actitudes positivas hacia el alumno, creencias en sus posibilidades e implicación en su enseñanza (gráfico 1).

De acuerdo con esa actitud, cualquier profesor o profesora, padre o madre deben considerar en el desarrollo educativo de los niños y niñas con CIL, algunos aspectos que personalicen su enseñanza. Así podrían expresarse que: a) La entidad diagnóstica no puede abstraerse de la persona, para que sea ésta el núcleo sustantivo. Si no se hace así, la adjetivación de limitación cognitiva se hace exclusiva, adquiriendo globali- 


\section{El término de capacidad \\ intelectual límite se abstrae de la \\ persona, nucleando toda la \\ explicación del individuo.}

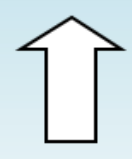

Realización de actividades con pérdida de significado, menor interés, no desarrollo de competencias.

\section{El profesorado genera una visión de limitación de inferioridad y pesimismo en las capacidades $y$ nees del alumno.}

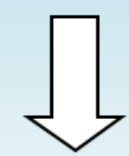

El desarrollo curricular y la aplicación de programas se conducen sobre objetivos y contenidos de menor nivel.

Gráfico 1. Evaluación de la capacidad intelectual límite y el desarrollo educativo. Adaptado de Luque y Luque-Rojas (2012).

dad explicativa (y negativa); b) El profesorado, padres y madres deben aceptar a su alumnado, hijos e hijas, conociendo sus limitaciones, pero también creyendo en una visión de potencialidad y desarrollo humanos; c) En los procesos de enseñanza-aprendizaje, en el desarrollo curricular y en la aplicación de programas, se deben conducir sobre objetivos y contenidos propios del currículum ordinario, orientados al desarrollo de estrategias y habilidades específicas susceptibles de generalización y aplicación a otros aprendizajes; d) Se ha de generar el interés, la significación y la motivación en actividades y aprendizajes en general, mejorando el desarrollo de competencias y de capacidades.

De acuerdo a esos aspectos, la enseñanza de este alumnado deberá contar a lo largo de sus etapas educativas (según su informe psicopedagógico) con los apoyos y recursos necesarios (cuadro 4).

Desde estas consideraciones, y con criterios operativos en el aula, a modo de pautas de actuación docente con el alumnado con CIL, podría seguirse dentro del esquema clásico del procesamiento de la información, una línea de trabajo que nos parece sugerente y productiva (gráfico 2).

De acuerdo con las características de su alumnado con CIL (una vez fijados sus puntos débiles) el profesor valorará los puntos fuertes y tratará de relacionarlos con los entornos de aprendizaje (aula, centro y comunidad). En este sentido, establecerá una base de posible actuación, planificación y desarrollo del currículum, ajustando para ello la metodología y las activi- dades. Finalmente, sobre este círculo de ejecución y rendimiento curriculares, deberá preocuparse además por su utilidad en relación a la práctica de actividades de la vida diaria y el desarrollo de competencias y habilidades.

Cuadro 4. Apoyos en el alumnado con $\mathrm{CIL}$

- Apoyo de refuerzo en el currículum.

- Apoyo específico en pedagogía terapéutica.

- Apoyo específico en audición y lenguaje.

- Apoyo específico en psicomotricidad.

- Apoyo específico en autonomía personal e independencia.

- Apoyo específico en la conducta y su regulación.

- Apoyo específico en las relaciones y habilidades sociales.

- Coordinación familia-centro-comunidad.

A través de los materiales de aula y programas específicos de intervención en:

- Desarrollo cognitivo.

- Desarrollo lingüístico.

- Desarrollo curricular.

- Estrategias de aprendizaje.

- En autonomía personal y habilidades sociales.

-...

Cualquier profesor, ante un alumno con CIL no solo ha de preguntarse cuáles son las tareas en las que tiene 


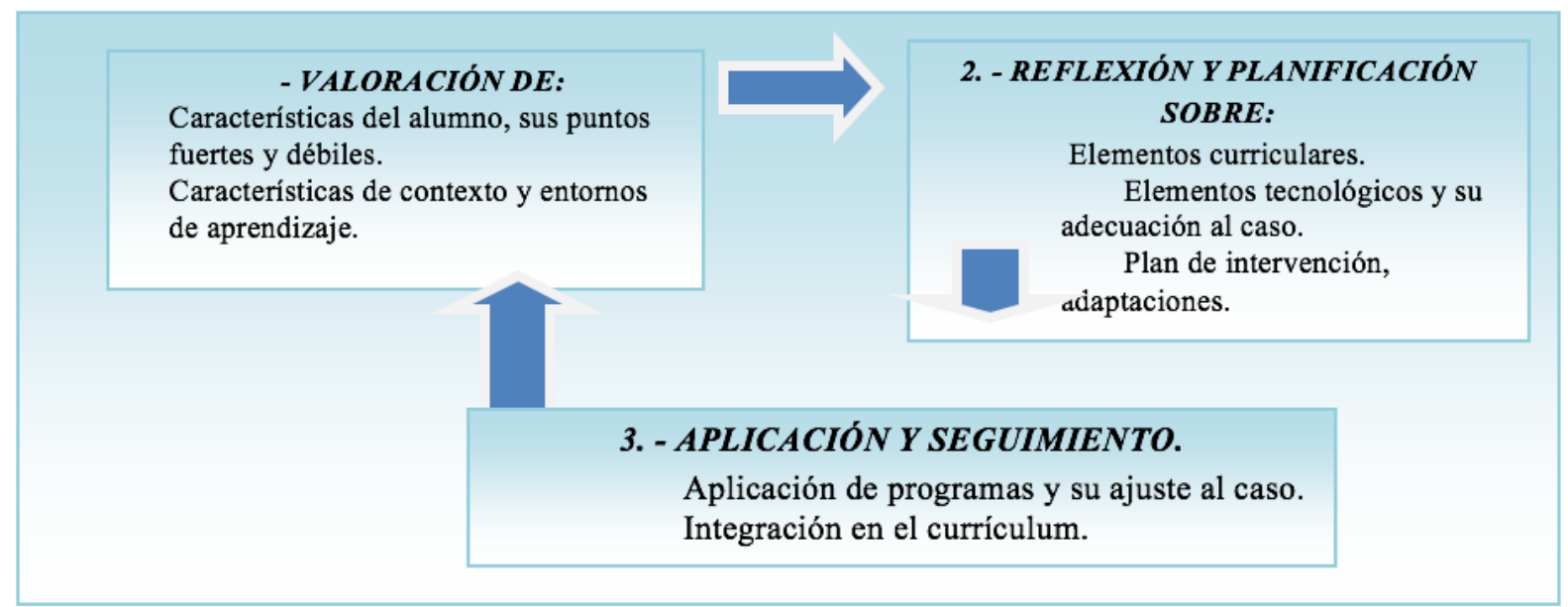

Gráfico 2. Pasos para un trabajo docente (Luque \& Luque-Rojas, 2012).

limitaciones para aprender o los contenidos y objetivos en la planificación de su proceso de instrucción, sino que habrá valorado también la competencia curricular del alumno (qué puede aprender como los demás) y qué puede aprender con ayuda y cómo aprende (estilos de aprendizaje). De esta forma, podrá establecer los programas ajustados al alumno y sus características, observar el desarrollo y ejecución dentro del currículum del aula y evaluar su grado de cumplimiento. Todo ello tiene cabida en el marco y diseño de la adaptación curricular, entendida como estrategia o instrumento de planificación y actuación docente, que hace operativa la respuesta a las necesidades educativas del alumno en concreto y la individualización de su proceso de enseñanza-aprendizaje (Luque \& Romero, 2002). Adaptación en la que, sin entrar en un análisis de su significatividad, se propondrán los recursos y adecuaciones del currículum que respondan a su situación personal y de aprendizaje, modificándose los elementos de ese currículum (salvo objetivos y contenidos nucleares) y no conllevando, desde la perspectiva del sistema educativo, un alejamiento del currículum general para etapa o ciclo. En este sentido, el profesor ante su alumno con CIL tiene el mismo reto que con cualquier otro alumno, tenga o no dificultades, el de adecuarse a sus necesidades específicas y lograr la integración de su atención en la planificación de aula o área.

Dadas las dificultades de aprendizaje y las consecuentes necesidades específicas del alumnado con CIL, los programas de intervención han de integrarse en la planificación y desarrollo curriculares, solo así ganan en ajuste y eficacia en el alumno, siendo además un mecanismo de compensación a sus necesidades, sin que ello suponga elementos de diferenciación discriminadora por sus dificultades. Con ello nos distanciamos de una aplicación parcial centrada en el déficit, en la que los programas de intervención específicos corren el riesgo de generar actuaciones descontextualizadas o fuera del currículum ordinario. Es en estas últimas instancias donde el alumnado presenta dificultades y se conforma un tratamiento singular (entrenamiento rehabilitador y exclusivo de una materia) y apartado del resto de la clase. Dada su necesidad y uso, la aplicación de programas de intervención psicopedagógica en el aula debe siempre apreciarse desde la acción tutorial. A esta la sigue y complementa una acción especializada o de apoyo y ello sin perder la perspectiva referencial de la propia acción tutorial que unifica y coordina los elementos de la enseñanza y del aprendizaje del alumnado.

La integración de los programas y, en general, de cualquier actividad que se realice con los alumnos con CIL persigue su ajuste y eficacia sobre el alumno. Es además un mecanismo de compensación a sus necesidades, sin que ello suponga elementos de diferenciación discriminadora por sus dificultades. Desde su necesidad y uso, la aplicación de programas de intervención psicopedagógica en el aula debe estar dentro de la acción tutorial, a la que sigue y complementa una acción especializada o de apoyo, sin perder la perspectiva referencial de aquella que unifica y coordina los elementos de la enseñanza y del aprendizaje del alumnado.

Desde una perspectiva de planificación educativa, los programas se pueden clasificar en programas de refuerzo académico (instrumentales o de áreas) y en programas de intervención específicos (cuadro 5), en función del ajuste curricular a las necesidades educativas del alumnado (Luque, 2006). En los niños y niñas con CIL, sin desdeñar los programas de refuerzo (con su generalidad de aplicación en áreas del currículum y aspectos instrumentales de lectura, expresión escrita, cálculo y razonamiento...), se dará mayor ajuste curricular a los de carácter específico, en tanto que su intervención es más centrada en el individuo y el área, con un objetivo básicamente de modificación o de actuación exclusiva en el desarrollo de habilidades o aptitudes. 
Cuadro 5. Programas (agrupación en función del ajuste curricular).

\begin{tabular}{|c|c|}
\hline Criterios prácticos de selección & Programas \\
\hline 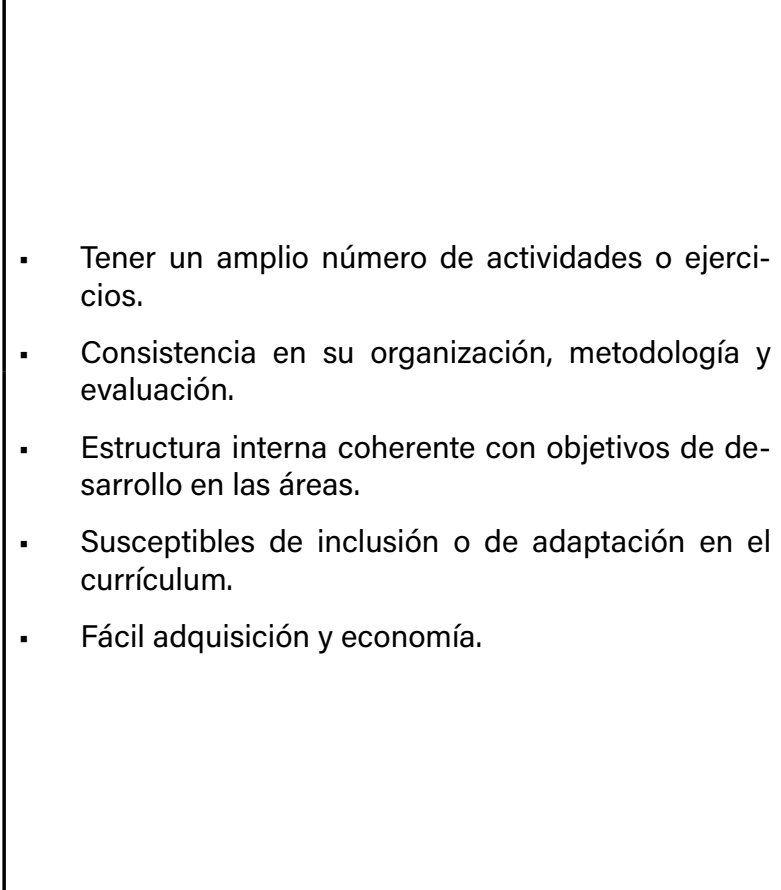 & \begin{tabular}{l} 
Programas de Refuerzo \\
- $\quad$ Reestructuración mínima de contenidos. \\
- $\quad$ Redistribución de actividades. \\
Temporalidad y metodología ajustadas al alumna- \\
do (mayor individualización). \\
- Consecución de objetivos generales de aula. \\
Problemas Escolares y de Bajo Rendimiento. \\
\multicolumn{1}{c}{ Programas Específicos } \\
- Reestructuración de contenidos en progresión y \\
complejidad de las dificultades individuales. \\
Actividades y metodología individualizadas. \\
- Actuación sobre áreas específicas (atención, per- \\
cepción, memoria, ...) \\
Consecución de objetivos en una temporalidad \\
mayor al resto del aula. \\
Dificultades Específicas de Aprendizaje, Trastorno por \\
Déficit de Atención con Hiperactividad y Capacidad \\
Intelectual Límite.
\end{tabular} \\
\hline
\end{tabular}

Nota: Tomado de Luque y Rodríguez (2006).

Este ajuste implicaría una reestructuración de contenidos de mayor complejidad en orden a las dificultades individuales, girando las actividades y metodología en torno a las áreas o aspectos de la atención, percepción, memoria, razonamiento, lectura comprensiva, estrategias cognitivas, etc. Así pues, se hace preciso considerar los criterios, estrategias docentes y actividades para la integración de esos programas en el desarrollo curricular del alumno (cuadro 6).

Finalmente, debe recordarse que la respuesta del alumnado con $\mathrm{CIL}$ a los programas de intervención obtendrá un mayor índice de eficacia y de integración socioeducativa si se aplican de forma coordinada por el equipo docente. Adicionalmente, deben contar con un diseño estructurado y organizado de programas cognitivos e instrumentales (estrategias cognitivas y de aprendizaje), una intervención en lo personal (autoestima y autoconcepto) y en lo social (habilidades sociales y de adaptación) y el oportuno protocolo de coordinación familia-centro.

\section{Discusión}

Existe acuerdo en describir la generalidad de casos de niños y niñas con CIL asociados o no a trastornos específicos (TDAH, lectura y escritura, matemáticas...) o de desarrollo, a partir de un funcionamiento cognitivo menos eficaz o de procesamiento más lento, así como con alguna limitación o disfunción en la capacidad de adaptación; todo ello explicado sobre una disfunción neurológica, como base de las alteraciones en el desarrollo de funciones psicológicas directamente implicadas en el aprendizaje. De acuerdo con esta conceptualización podríamos hacer algunas consideraciones para la reflexión.

Todas las actuaciones y procesos de estudio de los alumnos deben estar orientados a la inclusión social y educativa desde criterios de normalización, reduciendo la marca de un diagnóstico y favoreciendo la aceptación e integración personal y social. En todo ello, el profesorado debe considerar que su acción tutorial y la del equipo docente suponen el marco idóneo para el mejor conocimiento de la persona y de su desarrollo educativo.

La evaluación psicopedagógica del alumnado con CIL debe hacerse sobre un análisis profundo y riguroso de sus características personales y de contexto, de sus necesidades educativas y de las propuestas de intervención. En este sentido, podría ser preferible mantenerse en un estado de diagnóstico diferencial o de trastorno (general o específico), dando posibilidades de intervenciones correctoras y tiempos de observación que aseguren un diagnóstico más definitivo.

Los resultados de la evaluación psicopedagógica han de convertirse en necesidades específicas de apoyo educativo, lo que además de un cambio terminológico esperanzador, supone un ajuste al alumno sin perder 
de vista sus contextos (curricular, académico, social) proporcionándole una respuesta personalizada.

La intervención psicopedagógica y la respuesta específica a las necesidades de este alumnado, se integrará dentro del desarrollo curricular ordinario, con los ajustes y la adaptación que se consideren, persiguiendo objetivos de máximo desarrollo personal, intelectual, social y emocional.

Recordemos que, en un ámbito educativo inclusivo y en la diversidad del alumnado, los niños y niñas con CIL son tan iguales en su pertenencia como distintos en su individualidad por lo que podrán precisar apoyos específicos a su singularidad, aunque siempre dentro de las necesidades educativas que comparten con el resto de sus iguales. Por lo tanto, dado que el sistema educativo favorece la adecuación y ajuste pedagógicos, solo queda por resaltar que en el niño o niña con $\mathrm{CIL}$, como cualquier otro, debe ser considerado a partir de procesos de enseñanza-aprendizaje coherentes con sus circunstancias personales y sociales. Estos procesos deben perseguir el desarrollo de sus capacidades, intereses y motivaciones, y favorecer la satisfacción de sus necesidades educativas.

Señalemos, finalmente, la necesidad de continuar en el estudio de las personas con esta valoración diagnóstica no solo desde una perspectiva estructural de la inteligencia (definida con el término capacidad intelectual límite), sino desde su análisis y revisión en términos de funcionamiento intelectual (Borderline Intellectual Functioning). Con ello, a modo de recomendaciones para próximos trabajos, podría abundarse en investigaciones sobre desarrollo cognitivo y pensamiento estratégico.

\section{Referencias}

Alloway, T. (2010). Working memory and executive function profiles of individuals with borderline intellectual functioning. Journal of Intellectual Disability Research, 54(5), 448-456. doi:10.1111/j.1365-2788.2010.01281.x

American Psychiatric Association. (2002). Manual Diagnóstico y Estadístico de los Trastornos Mentales, 4a Revisión. Barcelona: Masson.

American Psychiatric Association. (2014). Manual Diagnóstico y Estadístico de los Trastornos Mentales, 5a Revisión. Barcelona: Masson.

Anderson, M. (1992). Intelligence and development: A cognitive theory. Oxford: Blackwell.

Artigas-Pallarés, J. (2003). Perfiles cognitivos de la inteligencia límite. Fronteras del retraso mental. Revista de Neurología, 36(Supl 1), 161-167. Recuperado de http://www. marthadebayle.com/wp-content/uploads/2013/09/ os10161.pdf

Artigas-Pallarés, J., Rigau-Ratera, E. \& García-Nonell, C. (2007). Relación entre capacidad de inteligencia límite y trastornos del neurodesarrollo. Revista de $\mathrm{Neu}$ - rología, 44(12), 739-744. Recuperado de http://www. neurologia.com/pdf/Web/4412/x120739.pdf

Bonifacci, P. \& Snowling, M. (2008). Speed of processing and reading disability: A cross-Linguistic investigation of dyslexia and borderline intellectual functioning. Cognition, 107(3), 999-1017. doi:10.1016/j.cognition.2007.12.006

Catts, H., Gillispie, M., Leonard, L., Kail, R. \& Miller, C. (2002). The role of speed of processing, rapid naming, and phonological awareness in reading achievement. Journal of Learning Disabilities, 35(6), 510-525. doi:10.1 177/00222194020350060301

El Justicia de Aragón. (2008). Estudio sobre las personas con capacidad intelectual límite. Recuperado de http:// www.eljusticiadearagon.com/gestor/ficheros/ $\underline{\text { n003618 CIL.pdf }}$

Elósegui, E., Luque, D. \& Casquero, D. (2011). Comportamiento de la Escala de Inteligencia para Niños de Wechsler-IV, en niños con capacidad intelectual límite. Trabajo presentado en el VI Congreso Internacional de Psicología y de Educación, Valladolid, España.

Elósegui, E., Luque, D. \& Casquero, M. (septiembre, 2012). Relación entre variables implicadas en el aprendizaje lectoescritor y la capacidad intelectual límite. Trabajo presentado en el XXI Congreso Internacional de Dificultades de Aprendizaje de la Universidad de Oviedo y Learning Disabilities World Wide Association LDW, Oviedo.

Greenspan, S. \& Love, P. (1997). Social intelligence and developmental disorder: Mental retardation, learning disabilities, and autism. En W. MacLean (Ed.), Ellis' handbook of mental deficiency, psychological theory, and research (pp. 311-342). Mahwah: Lawrence Eribaum.

Greenspan, S., Switzky, H. \& Granfield, J. (1996). Everyday intelligence and adaptative behaviour: A theoretical framework. En J. Jakobson \& J. Mulick (Eds.), Manual of diagnosis and professional practice in mental retardation (pp. 127-135). Boston: Addison-Wesley.

Haier, R., White, N. \& Alkire, M. (2003). Individual differences in general intelligence correlate with brain function during nonreasoning tasks. Intelligence, 31, 429-441. doi:10.1016/S0160-2896(03)00025-4

Hartman, E., Houwen, S., Scherder, E. \& Visscher, C. (2010). On the relationship between motor performance and executive functioning in children with intellectual disabilities. Journal of Intellectual Disability Research, 54(5), 468-477. doi:10.1111/j.1365-2788.2010.01284.x.

Jensen, A. (1998). The $g$ factor: The science of mental ability. Westport: Praeger Publishers.

Ley Orgánica (2/2006). "Ley Orgánica de la Educación (LOE) 2/2006". Boletín Oficial del Estado, Jefatura del Estado. Recuperado de https://www.boe.es/buscar/ pdf/2006/BOE-A-2006-7899-consolidado.pdf

Luque, D. (2006). Orientación Educativa e Intervención Psicopedagógica en el alumnado con discapacidad. Análisis de casos prácticos. Málaga: Aljibe.

Luque, D. (2009). Las necesidades educativas especiales 
como necesidades básicas. Una reflexión sobre la inclusión educativa. Revista Latinoamericana de Estudios Educativos, 39(3-4), 201-223. Recuperado de http://www.redalyc.org/pdf/270/27015078009.pdf

Luque, D. \& Rodríguez, G. (2006). Dificultades en el Aprendizaje: Unificación de Criterios diagnósticos (Vol. 3). Criterios de intervención pedagógica. Sevilla: Consejería de Educación y Junta de Andalucía.

Luque, D. \& Romero, J. (2002). Trastornos del desarrollo y Adaptación Curricular. Málaga: Aljibe.

Luque, D. \& Luque-Rojas, M. (2012). Aspectos Psicoeducativos en las relaciones de las TIC y la discapacidad intelectual. Revista Intercontinental de Psicología y Educación, 14(1), 27-48. Recuperado de http://www. redalyc.org/pdf/802/80224034003.pdf

Luque, D. \& Luque-Rojas, M. (2015). Evaluación psicopedagógica en la capacidad intelectual límite: Análisis de un perfil de WISC. Revista AOSMA, 19, 53-64. Recuperado de https://issuu.com/revista aosma/docs/ aosma19? $=15096006 / 10901348$

Luque, D., Elósegui, E. \& Casquero, D. (2014). Análisis del WISC-IV en una muestra de alumnos con capacidad intelectual límite. Revista de Psicología, 23(2), 14-27. doi:10.5354/0719-0581.2014.36145

Morgado, I. (2005). Psicobiología del aprendizaje y la memoria: Fundamentos y avances recientes. Revista de Neurología, 40(5), 289-297. Recuperado de https:// extension.uned.es/archivos publicos/webex actividades/5376/aprymemrn20053.pdf

Morton, J. (2004). Understanding developmental disorders: $A$ causal modelling approach. Oxford: Blackwell.

Narbona, J. \& Crespo-Eguílaz, N. (2005). Trastornos de memoria y de atención en disfunciones cerebrales del niño. Revista de Neurología, 40(Supl 1), 33-36. Recuperado de http://www.neurologia.com/pdf/Web/40S1/sS1S033.pdf

Pallisera, M. (2003). Capacidad intelectual límite e integración laboral: desde la relatividad conceptual al análisis de las experiencias integradoras. Bordón, Revista de Pedagogía, 55(4), 555-568.
Romero, J. \& Lavigne, R. (2005). Dificultades en el Aprendizaje. Unificación de criterios diagnósticos (Vol. 1). Definición, características y tipos. Sevilla: Consejería de Educación y Junta de Andalucía.

Shaw, S. (2008). An Educational Programming Framework for a Subset of Students With Diverse Learning Needs: Borderline Intellectual Functioning. Intervention in School and Clinic, 43(5), 291-299. doi:10.1177/1053451208314735

Schuchardt, K., Gebhardt, M. \& Mäehler, C. (2010). Working Memory functions in children with differents degrees of intellectual disability. Journal of Intellectual Disability Research, 54(4), 346-353. doi:10.1111/j.13652788.2010.01265.x.

Singh, V. (2004). Education of the slow learners. New Delhi: Sarup.

Sternberg, R. (1988). The triarchic mind: A new theory of human intelligence. Nueva York: Penguin.

Tirapu-Ustárroz, J. \& Muñoz-Céspedes, J. (2005). Memoria y funciones ejecutivas. Revista de Neurología, 41(8), 475-484. Recuperado de http://www.neurologia. com/pdf/web/4108/t080475.pdf

Trianes, M. \& Luque, D. (2013). Manual de Psicología de la Educación para Psicólogos. Málaga: Universidad de Málaga.

Verguts, T. \& DeBoeck, P. (2001). On the correlation between working memory capacity and performance on intelligence tests. Learning and Individual Differences, 13(1), 37-56. doi:10.1016/S1041-6080(02)00049-3

Vuijk, P., Hartman, E., Scherder, E. \& Visscher, C. (2010). Motor performance of children with mild intellectual disability and borderline intellectual functioning. Journal of Intellectual Disability Research, 54, 955-965. doi:10.1111/j.1365-2788.2010.01318.x. 